\title{
The Rajarata Pregnancy Cohort (RaPCo): study protocol
}

Thilini Chanchala Agampodi ${ }^{*}$ D , Nuwan Darshana Wickramasinghe ${ }^{1}$, Rampathige Indika Ruwan Prasanna ${ }^{2}$, Malawara Kankanamalage Lasandha Irangani ${ }^{3}$, Jayasundara Mudiyanselage Samarakoon Banda ${ }^{4}$, Pradana Mudiyanselage Bandula Jayathilake ${ }^{5}$, Ayesh Hettiarachchi ${ }^{1}$, Gayani Amarasinghe ${ }^{1}$, Imasha Jayasinghe ${ }^{1}$, Iresha Koralagedara ${ }^{6}$, Sajaan Praveena Gunarathne ${ }^{1}$, Sujanthi Wickramage7, Janith Warnasekara ${ }^{1}$, Niroshan Lokunarangoda ${ }^{8}$, Vasana Mendis ${ }^{9}$, Ajith Kumara Dissanayaka ${ }^{10}$, Jagath Premadasa ${ }^{11}$, Nandana Hettigama ${ }^{10}$, Dayaratne Koralagedara ${ }^{11}$, Manjula Weerasinghe ${ }^{1}$, Krishanthi Malawanage $^{12}$, Hemali Jayakodi ${ }^{13}$, Anuprabha Wickramasinghe ${ }^{14}$ and Suneth Buddhika Agampodi ${ }^{1}$

\begin{abstract}
Background: Ending preventable maternal deaths remains a global priority and in the later stages of obstetric transition, identifying the social determinants of maternal health outcomes is essential to address stagnating maternal mortality rates. Countries would hardly achieve the Sustainable Development Goal (SGD) targets on maternal health, unless the complex and context-specific socio-economic aetiologies associated with maternal mental health and suicide are identified. The Rajarata Pregnancy Cohort (RaPCo) is a prospective cohort study, designed to explore the interactions between social determinants and maternal mental health in determining pregnancy and new-born outcomes.
\end{abstract}

Methods: The study will recruit all eligible pregnant women in the maternal care programme of Anuradhapura district, Sri Lanka from July to September 2019. The estimated sample size is 2400 . We will assess the sociodemographic and economic status, social capital, gender-based violence and mental health, including a clinical examination and biochemical investigations during the first trimester. Participants will undergo four follow-ups at 2nd and 3rd trimesters, at delivery and in early postpartum. The new-borns will be followed up at birth, neonatal period, at 6 six months and at 1 year. Pregnancy and child outcome data will be collected using direct contact. Qualitative studies will be carried out to understand the complex social factors and behavioural dimensions related to abortion, antenatal depression, maternal deaths and near misses.

(Continued on next page)

\footnotetext{
* Correspondence: thilinichanchala@yahoo.com

'Department of Community Medicine, Faculty of Medicine and Allied

Sciences, Rajarata University of Sri Lanka, Anuradhapura, Sri Lanka

Full list of author information is available at the end of the article
}

(c) The Author(s). 2020 Open Access This article is licensed under a Creative Commons Attribution 4.0 International License, which permits use, sharing, adaptation, distribution and reproduction in any medium or format, as long as you give appropriate credit to the original author(s) and the source, provide a link to the Creative Commons licence, and indicate if changes were made. The images or other third party material in this article are included in the article's Creative Commons licence, unless indicated otherwise in a credit line to the material. If material is not included in the article's Creative Commons licence and your intended use is not permitted by statutory regulation or exceeds the permitted use, you will need to obtain permission directly from the copyright holder. To view a copy of this licence, visit http://creativecommons.org/licenses/by/4.0/. The Creative Commons Public Domain Dedication waiver (http://creativecommons.org/publicdomain/zero/1.0/) applies to the data made available in this article, unless otherwise stated in a credit line to the data. 
(Continued from previous page)

Discussion: This is the first reported maternal cohort in Sri Lanka focusing on social determinants and mental health. As a country in stage four of obstetric transition, these findings will provide generalizable evidence on achieving SGD targets in low- and middle-income countries. The study will be conducted in a district with multicultural, multi-ethnic and diverse community characteristics; thus, will enable the evidence generated to be applied in many different contexts. The study also possesses the strength of using direct participant contact, data collection, measurement, examination and biochemical testing to minimise errors in routinely collected data. The RaPCo study will be able to generate evidence to strengthen policies to further reduce maternal deaths in the local, regional and global contexts particularly focusing on social factors and mental health, which are not optimally addressed in the global agenda.

Keywords: Pregnancy, Cohort, Sri Lanka, Social determinants, Mental health

\section{Background}

Every day, an average of 830 pregnant women around the globe die due to preventable causes [1]. Despite the drastic reduction in maternal mortality by $45 \%$ during the period of 1990-2015 [2], the global maternal health agenda is still facing major challenges and Ending Preventable Maternal Mortality (EPMM) is far from the reach [3]. The Millennium Development Goal (MDG) target of achieving annual rate of reduction (ARR) in maternal mortality ratio (MMR) of $5.5 \%$ was not a success. However, this target pushed the countries around the world to increase ARR from $1.1 \%$ in $1990-2000$ period to $4.1 \%$ in $2000-2010$ period [4]. Currently, the Sustainable Development Goal (SGD) 3 targets to reduce maternal mortality in the world to $70 / 100000$ live births [3].

\section{Maternal health and social capital}

With the "obstetric transition", causes of maternal deaths are shifting from communicable diseases and direct obstetric causes such as sepsis, haemorrhage, pulmonary embolism (phase 1) to non-communicable diseases and indirect causes [5] (phase 4 and 5). Highincome countries as well as some of the middle-income countries are in phase four and the shift is clearly demonstrated in the most recent maternal death estimates [6]. However, further reduction of maternal deaths has been a challenge and stagnation of MMRs is observed at global level [7].

Social development is identified as a prime to further reduce MMR in the latter phases of obstetric transition. Yet, the global maternal health agenda is primarily focused on medical interventions, neglecting the effects of social determinants and social interventions as root causes of all proximal determinants. Some aspects of social determinants, such as social capital in pregnancy and postpartum, are discussed only in few instances in global literature. "Social capital" in general reflects the state of social interactions among people. It is defined as the "features of social organization, such as trust, norms and networks that can improve the efficiency of society by facilitating coordinated actions" [8]. Social capital is a relatively new concept widely used in the field of social sciences $[9,10]$. More recently, with the rise in global attention towards social determinants of health, social capital is considered as an important determinant that can influence the health of individuals and communities [11-14]. Few available studies on social capital and maternal health show that high social capital during pregnancy is associated with higher levels of self-rated health [11], lower levels of postpartum psychosis [15] and health related behaviours [16]. Social capital is also associated with health related behaviours in pregnancy [17]. It is identified that social capital in pregnancy is unique and it mainly encompasses cognitive and structural bonding in the immediate micro community of pregnant women. It is also hypothesized that these strong social ties exert a considerable impact on psychosocial wellbeing of pregnant women and having a significant inverse relationship to antenatal and postpartum anxiety and depression [17]. Micro-geographical variations of social capital is observed through ecological studies and may partly explain the health inequalities [18]. However, the predictive ability of social capital on pregnancy outcome has not yet been assessed.

\section{Maternal mental health and suicides}

Globally, maternal suicide has emerged as a leading cause of maternal deaths over the past few decades [19]. It has become a leading cause of maternal deaths in some countries [20-22]. Suicide is only the tip of the iceberg. A recent systematic review shows that the suicidal ideation is largely underreported and the available tools are not capturing the actual risk [23]. Pregnancy and childbirth represent a time of increased vulnerability, during which a woman is exposed to many physiological and psychosocial changes, which put pregnant and postpartum women at increased risk of mental health problems. It has been consistently shown that the prevalence of maternal mental health issues are much greater in low- 
income countries (LIC) and lower-middle-income countries (LMIC) (16\% and 20\%, respectively) [24] than in high-income countries (HIC) [25]. The variability in estimates, seen especially in LIC and LMIC, is due to the differences in diagnostic criteria and timing of screening [26], socio-cultural and biological factors [27], and availability of data [25]. Of the mental health problems experienced by pregnant and postpartum women, antenatal depression, anxiety, and post-partum depression are the most common [28].

Antenatal depression, maternal anxiety and lack of psychosocial support during pregnancy is associated with low birth weight (LBW)/preterm birth (PTB), reduced gestational age at birth or intra uterine growth retardation (IUGR) [29-31]. Maternal depression has long-term effects on child development [32], poor mother-infant interaction [33], negative effects in the infant [34], and problems with cognitive development [35]. It is also shown that affective disorders [36] and attention-deficit/hyperactivity disorder (ADHD) are more common among children and adolescents of depressed mothers [37]. Multiple investigations have also found that women who experience antenatal depression are more likely to experience postpartum depression (PPD) as well [38-40].

\section{Sri Lankan scenario}

In Sri Lanka, reanalysis of maternal deaths using International Classification of Diseases for Maternal Mortality (ICD MM) [41], the ICD version recommended for causes of maternal deaths classification, showed that suicide is the leading cause of maternal deaths in this area [42]. Further, previous research showed that $16.2 \%$ [27] of pregnant women are having depression and anxiety, and $27.1 \%$ of postpartum women are having depression [43]. In this given context, to achieve EPMM, suicide prevention and maternal mental health promotion should be considered as a high priority in Sri Lankan public health settings. Tackling this problem needs evidence on what determines maternal mental health at local level. Even though the global evidence base provides an overview of determinants of maternal suicide, it is crucial to consider the cultural and context-specific determinants in order to effectively promote maternal mental health. Further, with a view of capturing the dynamics of health-related behaviours, it is important to generate evidence based on longitudinal studies rather than solely based on cross-sectional studies. The more recent studies on exploring social capital and maternal health provided extensive data on the probable effects of social capital on maternal mental health. So far, there are no reported objective evaluations of social capital and maternal mental health conducted using prospective study designs. For evidence informed decision-making, high quality data based on robust methodologies are required. The best evidence for this type of outcome assessments comes though cohort studies. The Sri Lankan health system is one of the best in LMIC in maternal care and it usually captures more than $95 \%$ of pregnant women through public health pregnancy registration system [44]. This system provides a good opportunity to establish community cohorts. The aim of the proposed study, which is the Rajarata Pregnancy Cohort (RaPCo), is to evaluate the possible associations and interactions of maternal mental health and maternal social capital with the pregnancy outcome, neonatal outcomes and early childhood health and development outcomes in the Anuradhapura district, in order to aid in developing evidence-based guidelines on EPMM.

\section{Methods \\ Aims}

1. To determine the aetiological associations of mental health during pregnancy with pregnancy outcomes, neonatal outcomes and early childhood health and development outcomes.

2. To determine the possible interactions of complex social issues, social capital, socio-economic position, personality and gender-based violence on healthrelated issues during pregnancy, pregnancy outcomes, neonatal outcomes and early childhood outcomes.

\section{Study hypotheses (Fig. 1)}

1. Social determinants such as poor social capital, poor socio-economic position and gender-based violence are independent risk factors of perinatal anxiety and depression.

2. Social capital of pregnant women is associated with anxiety and depression during pregnancy at both individual and ecological levels.

3. Mental health during early and late pregnancy is associated with pregnancy outcomes such as intra uterine growth retardation preterm labor and low birth weight.

4. Mental health during pregnancy and postpartum periods is a risk factor for non-exclusive breast feeding.

5. Mental health during pregnancy and postpartum periods is a risk factor for growth and development during infancy.

6. Social determinants such as poor social capital, poor socio-economic position and gender-based 


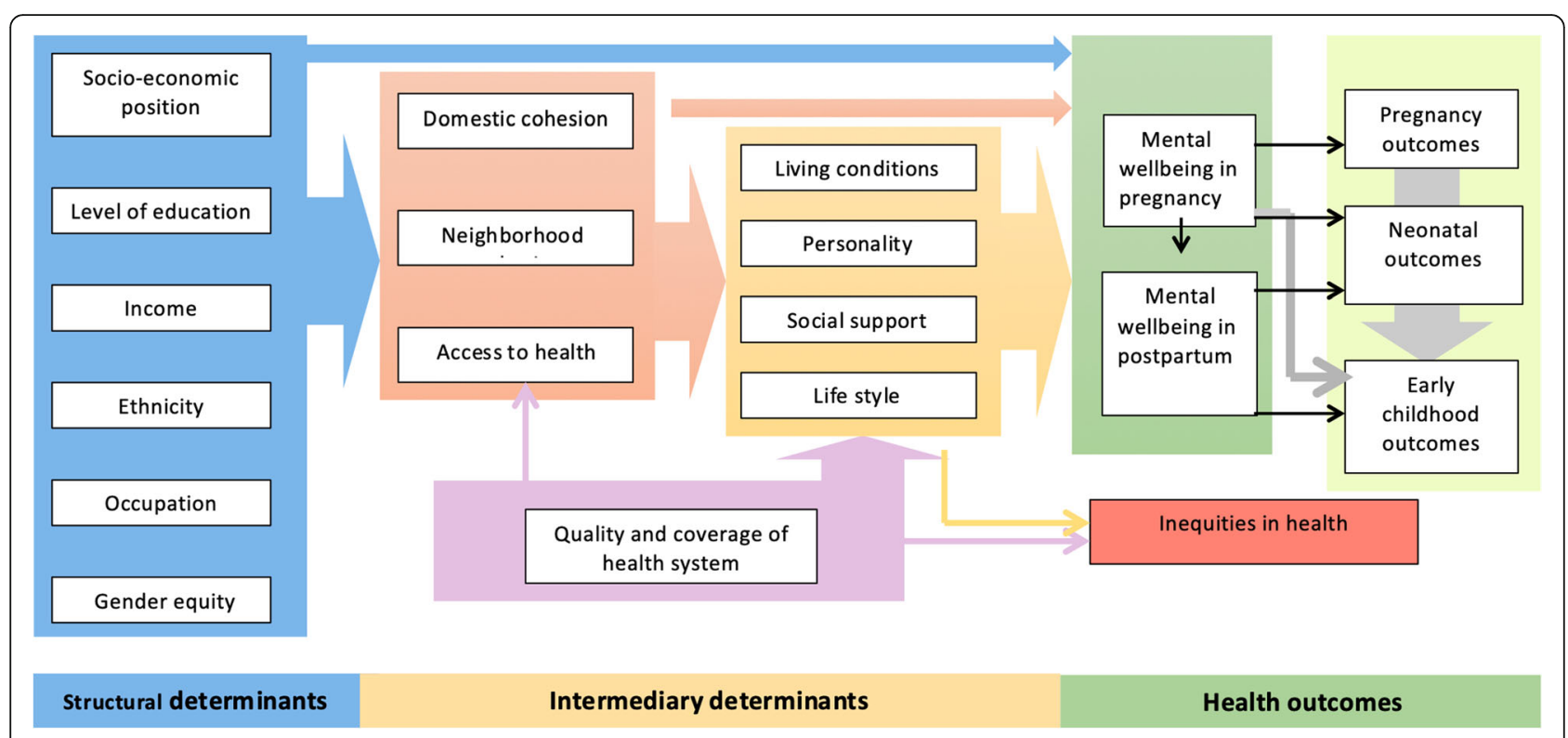

Fig. 1 Conceptual framework of RaPCo study hypothesis

violence are independent risk factors of pregnancy and early childhood outcomes.

7. Abortion is associated with subsequent anxiety and depression.

8. Health system defaults such as unmet need and failure to follow-up for risk conditions are associated with adverse perinatal outcomes.

9. Intra-district micro-geographical variations exist in social determinants of maternal health and influence perinatal outcomes.

10. Differentials in social determinants has a causative impact on inequity of maternal and early childhood health and wellbeing.

\section{Study design}

This study is a prospective cohort study of pregnant women. Pregnant women will be recruited to the study in their early pregnancy (before 12 weeks) where they will undergo baseline assessment and will be followed up in the second and third trimesters in the field clinics and at delivery in the hospitals. Women will be followed up in the postpartum period in the field and infants will be followed up at routine child welfare clinics up to 1 year of age.

\section{Study setting}

The proposed study will be carried out in the Anuradhapura district (Fig. 2), which is the largest district in Sri Lanka. According to the routinely reported data, the resident population is 902930 with a birth rate of 17.8 / 1000 population [45]. The total fertility rate is 2.4 and the median age at first birth is 23.9 years, which is one of the lowest in Sri Lanka [46]. The number of pregnant mothers registered in the area in 2015 was more than 17000 and of them, $82.3 \%$ registered in field clinics before the eighth week of pregnancy and $96.0 \%$ had at least one clinic visit before delivery. The number of live births was 15376.

The services for pregnant women are provided through medical officer of health $(\mathrm{MOH})$ and currently the district is divided in to $22 \mathrm{MOH}$ areas. $\mathrm{MOH}$ is a community physician delivering public health services to the respective residing population. The grass-root level maternal and child health services are delivered through public health midwifes (PHM). Each $\mathrm{MOH}$ is consisted of 8-24 PHM areas. The PHMs are supervised by the Public Health Nursing Sisters (PHNS) (one PHNS per $\mathrm{MOH}$ each area). The district as a whole is divided in to 275 PHM areas with each PHM area is having a population of 1500-4000 people. PHMs register all pregnant women in the respective PHM areas in the routine maternal care service in the country in a register known as "Pregnant Mothers' Register". They provide domiciliary care at home visits and clinic care at antenatal clinics for all pregnant women. Curative services are provided through 56 hospitals/primary care units, including one teaching hospital (tertiary care) and three base hospitals (secondary care). Almost all deliveries (99.5\%) are taken place in government hospitals [45].

\section{Study population}

The study population for this study will include all pregnant women residing in the Anuradhapura district. 


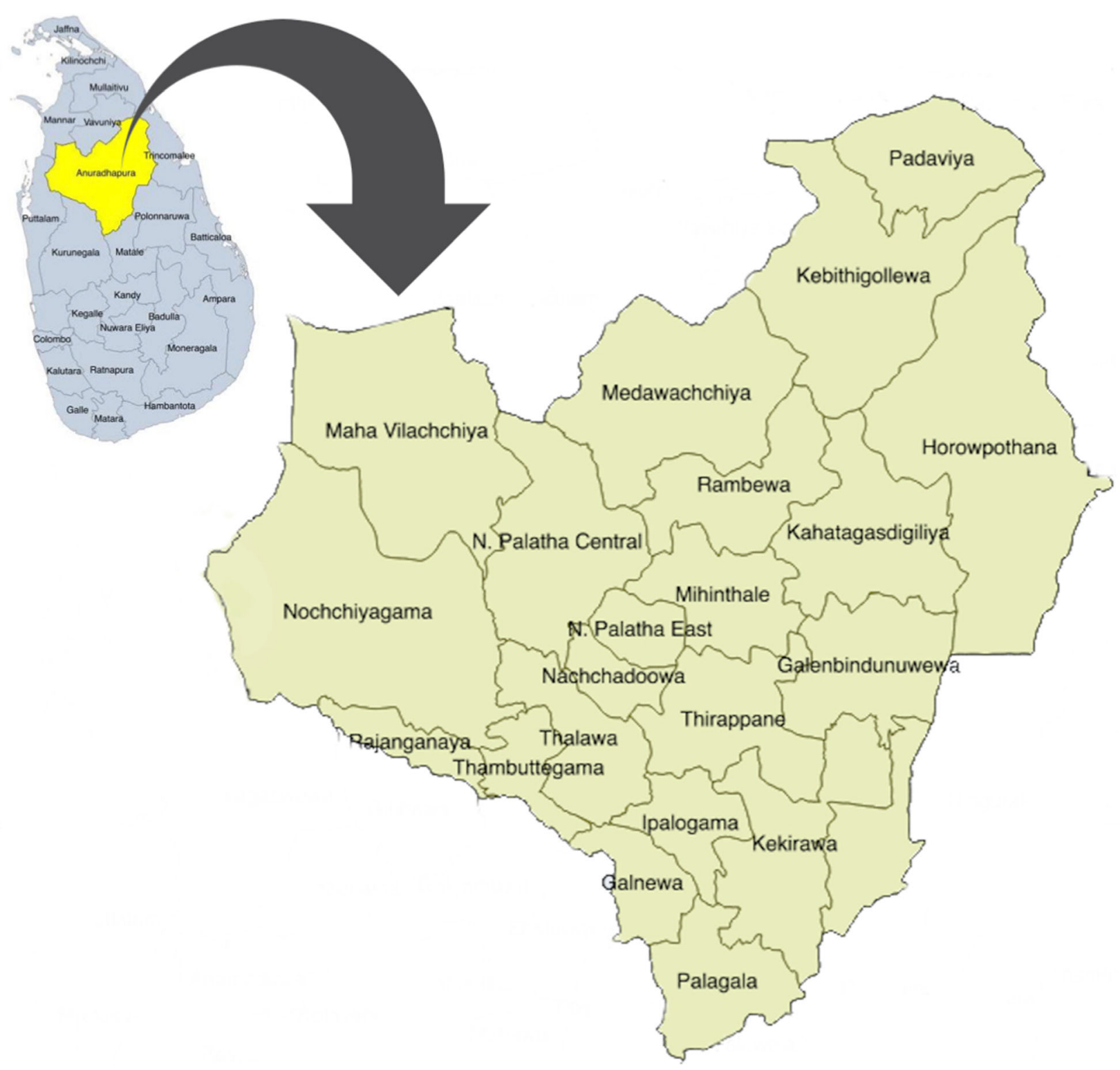

Fig. 2 Spatial distribution of 22 participant recruitment areas of RaPCo study in the Anuradhapura district, Sri Lanka

\section{Study sample}

All pregnant women registered in pregnant mothers' registers during the period of 1st July to 30th September 2019 in the maternal care programme in the Anuradhapura district will be recruited as the study participants.

\section{Inclusion criteria}

1. Pregnant women registered in pregnant mothers' register of PHM and have completed the booking visit (first clinic visit) in antenatal clinics in the Anuradhapura district.

2. Permanent residence in the Anuradhapura district during the year ahead.
3. Period of amenorrhoea (POA)/gestational age (GA) less than 12 weeks by the time of recruitment.

\section{Exclusion criteria}

1. Pregnant women planning to leave the study area for childbirth or after the childbirth.

\section{Sample size}

For the sample size calculation, we used a prevalence of antenatal depression of $16.2 \%$ as reported in Anuradhapura 2011 [27]. The relative risk [RR] of antenatal depression on for LBW, and IUGR reported in previous meta-analysis was 1.49 
[95\% CI $=1.25-1.77]$ and $1.45[95 \% \mathrm{CI}=1.05-2.02]$ respectively [47]. We used the lowest RR for this calculation.

We used the sample size formula for Cohort studies as given by Kelsy et al. [48].

$$
n_{1}=\frac{\left(Z_{\alpha / 2}+Z_{1 \beta}\right)^{2} \overline{p q}(r-1)}{r\left(p_{1}-p_{2}\right)^{2}}
$$

where, $\mathrm{n}_{1}=$ number of exposed, $\mathrm{n}_{2}=$ number of unexposed, $Z_{\alpha / 2}=$ standard normal deviate for two-tailed test, alpha level was taken as $90 \%, Z_{\beta}=$ standard normal deviate for one-tailed test based on beta level of $80 \%, r=r a-$ tio of unexposed to exposed 1: $0.16, \mathrm{p}_{1}=$ proportion of pregnant women with depression having a bad pregnancy outcome $(0.229), \mathrm{q}_{1}=1-\mathrm{p}_{1}, \mathrm{p}_{2}=$ proportion of healthy pregnant women with bad pregnancy outcome (0.17), $\mathrm{q}_{2}=1-\mathrm{p}_{2}, \quad \bar{p}=\frac{p_{1}+r p_{2}}{r+1}, \quad \bar{q}=1-\bar{p}$. The calculated minimal sample size is 1948. With an anticipated loss to follow-up of $20 \%$, the required size of the sample is computed to be 2338 . We will recruit all pregnant women within a period of three months starting from July 2019 and the estimated sample will be around 2400 .

\section{Recruitment of study participants}

RaPCo study flow chart (Fig. 3) and the participant timeline (Table 1) indicates the timing and procedures of the study from participant recruitment to close-out. The process of recruitment of participants will be mediated at the district level after explaining and informing the district level health authorities in order to initiate and maintain a strong baseline component. Advocacy meetings will be held to inform all $22 \mathrm{MOHs}$ and the PHNS at district level and for the PHMs at divisional level using the routine monthly public health conference gatherings. Advocacy procedures will include strategic and systematic procedures including project presentations, video clips and unique information leaflets to all stakeholders including MOHs, PHNSs, PHMs and pregnant women. The field PHMs will do the initial introduction to the eligible pregnant mothers about the study. Once the pregnant women are registered and have completed the booking visit, they will be offered a date and the venue for the first contact with the study team using an invitation to a special clinic. The clinic schedules and sites will be predetermined at district and divisional level and on the consensus of all public health authorities. Pregnant women will be given a special set of instructions to bring all previous medical records and also to attend the clinic with fasting for $8 \mathrm{~h}$ to conduct necessary blood investigations. A team of researchers and healthcare providers from the public health system will jointly conduct the special clinic. A team will consist of an MBBS qualified medical officer, two pre-intern medical officers, four second-year medical undergraduates trained on data collection and a trained nurse for drawing blood samples. Eligible pregnant women will be given an explanation on the study objectives and informed written consent will be sought before the recruitment. Once consented, initial registration to the cohort will be done. All routine sample collection for anaemia, grouping and $\mathrm{Rh}, \mathrm{VDRL}$, urine albumin and sugar will be done during this clinic. OGTT test will be performed for all pregnant women, who are not already having a diagnosis of diabetes mellitus. While waiting for the blood sample collection of the 2nd hour test, data collection and health education will be carried out. Fully trained interviewers will collect data from the participants. Clinical examination will be done by MBBS qualified medical practitioners during this clinic session.

\section{Baseline assessment}

Several questionnaires and laboratory investigations will be carried out (as outlined below) to assess the health status of pregnant mothers at baseline. Basic sociodemographic data, pregnancy details, medical and drug history will be obtained using an intervieweradministered questionnaire. Maternal mental health status, social capital, personality type, nutrition intake and economic costs of pregnancy will be assessed using selfadministered questionnaires. Other self-administered questionnaires will be handed over to the pregnant women to complete at home. These questionnaires will be collected during the next clinic visit due in the second trimester. We will use a bar-coding system for all study instruments and all self-administered questionnaires will be without the personal identification details, hence the confidentiality will be maintained. Personal identification details will be collected during the first interview and subsequently, only bar-coded tools will be used.

\section{Study tools}

We will use several study tools to assess the intended measures as described below.

1. Socio-demographic factors, medical history and pregnancy details: A study-specific intervieweradministered questionnaire prepared by the investigators in Sinhala and Tamil

2. Anxiety and Depression in pregnancy and postpartum: Validated Sinhala and Tamil versions of the Edinburgh Post-Partum Depression Scale (EPDS) [49]

3. Gender-based violence: Validated Sinhala and Tamil versions of Abuse Assessment Screen (AAS) [50]

4. Productivity cost due to maternal morbidities: Culturally adapted and validated Sinhala and Tamil 
Pregnat women registered in field antenatal clinics and the gestational age/ period of amenorrhoea less than 12 weeks

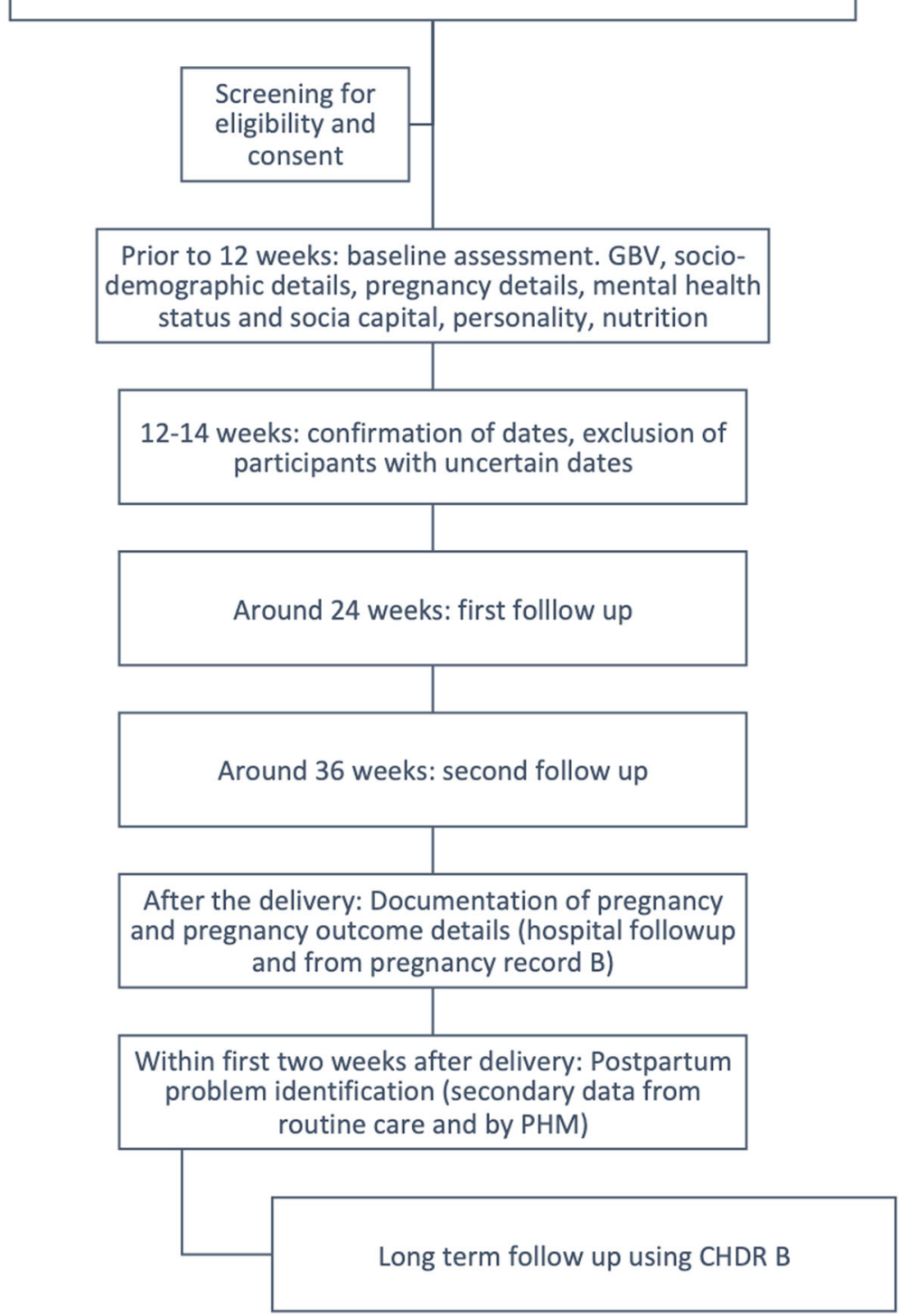

Fig. 3 RaPCo study flow chart

versions of the WHO Impact Tool Kit (selected components) [51]

5. Personality: Culturally adapted and validated Sinhala and Tamil versions of Ten Item Personality Inventory (TIPI) [52]

6. Social capital in pregnancy and postpartum: Low and middle income countries Social Capital Assessment Scale related to maternal Health (LSCAT-MH) (originally developed and validated Sinhala and Tamil versions) [53]

7. Dietary assessment: Food frequency questionnaire and assessment of diet plate prepared by the investigators
8. Psychological distress: Validated Sinhala and Tamil of General Health questionnaire 12 [54]

9. Experience based house hold food security: Translated and adapted Sinhala and Tamil versions of Latin American and Carribbean Household Food Security Scale (ELCSA) [55] (This questionnaire was translated according to Sumathipala and Murrays technique [56] and an expert validation was performed using a panel of experts in public health and nutrition (see additional files one and two).

Since the literacy rate is $94.8 \%$ in the study area [57], we expect that the pregnant mothers will be able to 
Table 1 Patient timeline of Rajarata Pregnancy Cohort

\begin{tabular}{|c|c|c|c|c|c|c|c|c|}
\hline \multicolumn{9}{|l|}{ STUDY PERIOD } \\
\hline \multirow[b]{2}{*}{ Time point } & \multirow{2}{*}{$\begin{array}{l}\text { Enrolment } \\
\text {-t1 6-8 w }\end{array}$} & \multirow{2}{*}{$\begin{array}{l}\text { Allocation } \\
\text { t0 } 12 \mathrm{w}\end{array}$} & \multicolumn{6}{|c|}{ Post allocation } \\
\hline & & & $\begin{array}{l}\text { t1 } \\
25- \\
28 \mathrm{~W}\end{array}$ & $\begin{array}{l}\mathrm{t} 2 \\
32- \\
36 \mathrm{w}\end{array}$ & $\begin{array}{l}\text { t3 } \\
\text { Delivery }\end{array}$ & $\begin{array}{l}\text { t4 } \\
\text { Postpartum }\end{array}$ & $\begin{array}{l}\text { t5 } \\
\text { One } \\
\text { year }\end{array}$ & $\begin{array}{l}\text { Close- } \\
\text { out }\end{array}$ \\
\hline Enrollment & $x$ & & & & & & & \\
\hline Eligibility Screen & $x$ & & & & & & & \\
\hline Informed consent & $x$ & & & & & & & \\
\hline Recruitment & & $x$ & & & & & & \\
\hline Socio-demographic information, gender-based violence, personality & & $x$ & & & & & & \\
\hline $\begin{array}{l}\text { Past obstetric history } \\
\text { Past Medical history }\end{array}$ & & $x$ & & & & & & \\
\hline $\begin{array}{l}\text { Obstetric details of current pregnancy, Medical complications of } \\
\text { present pregnancy, Mental Health, Income and expenditure }\end{array}$ & & $x$ & $x$ & $x$ & & & & \\
\hline Social capital & & $x$ & $x$ & & & & & \\
\hline Food security & & & & $x$ & & & & \\
\hline Maternal height, waist to hip ratio & & $x$ & & & & & & \\
\hline Maternal weight, general examination, cardio vascular variables & & $x$ & $x$ & $x$ & & & & \\
\hline $\begin{array}{l}\text { Liver function tests, Serum cholesterol, urine full report, blood picture, } \\
\text { High performance liquid chromatography, serum ferritin }\end{array}$ & & $x$ & & & & & & \\
\hline Blood glucose (OGTT) & & $x$ & $x$ & & & & & \\
\hline Hemoglobin, red cell indices & & $x$ & $x$ & & & & & \\
\hline Serum Cortisol & & & $x$ & & & & & \\
\hline $\begin{array}{l}\text { Mode of delivery, birth weight, maternal complications, neonatal } \\
\text { complications }\end{array}$ & & & & & $x$ & & & \\
\hline Details of breastfeeding & & & & & $x$ & $x$ & & \\
\hline Postpartum depression, loneliness, neonatal details & & & & & & $x$ & & \\
\hline Growth and development indicators & & & & & & & $x$ & \\
\hline Close out & & & & & & & & $x$ \\
\hline
\end{tabular}

complete the self-administered questionnaires. However, if there are any mothers having difficulties in completing the questionnaires on their own, data collectors will assist them in the procedure. Data extraction at follow-up visits will be done using data extraction forms prepared by the investigators.

\section{Measurements and clinical examination}

Anthropometric measurements will be collected using the standard protocols [58]. We will measure weight, height, waist and hip circumference during the clinic visit. Blood pressure measurement and the to pulse rate will be recorded using automated BP monitors. A general examination and auscultation of heart will be done using clinical examination guidelines and specific cardiac stethoscopes.

\section{Biochemical tests}

All routine biochemical analysis conducted at antenatal clinics will be offered through the RaPCo, except VDRL and grouping and $\mathrm{Rh}$, which will be done in the reference hospitals in the Sri Lankan maternal health programme. The venepuncture will be done by an experienced, registered nurse using aseptic techniques and standard precautions, as a part of routine booking visit sample collection. Baseline biochemical parameters will include full blood count, plasma glucose, liver function tests, serum cholesterol and urine full report. A sample of whole blood, serum and urine will be collected. Samples will be immediately sent to the Public Health Research Laboratory of Faculty of Medicine and Allied Sciences, Saliyapura. Full blood count and plasma glucose levels will be done on the same day. A sample of urine will also be collected to a sterile bottle. Urine analysis will be done using 10 parameter strip test. An aliquot of whole blood, serum and processed urine will be stored at $-80^{\circ} \mathrm{C}$ for batch-wise analysis.

\section{Follow-up visits}

First follow-up visit (second trimester of pregnancy)

All pregnant women will be followed up in routine antenatal clinics/antenatal sessions of the Sri Lankan 
maternal care programme. Those who are not attending the clinics or antenatal sessions will be followed up at home. The first RaPCo follow-up visit will be done around 24 weeks of gestation (during the second trimester). All data pertaining to the pregnancy after the first contact will be obtained from the pregnancy record maintained in the maternal care system (H519 A portion) and an interviewer-administered questionnaire. In the second trimester, we will reassess pregnancy weight gain, blood glucose, haemoglobin level, social capital, mental health and health expenditure. Serum cortisol levels will be assessed in a sub-sample of women.

\section{Second follow-up visit (third trimester of pregnancy)}

Second follow-up visit will be similar to the first followup visit. This will be done around 32-36 weeks of gestation. Maternal mental health, food security, health expenditure and blood biochemistry related to the project objectives will be assessed during this visit.

\section{Third follow-up (hospital follow up at delivery)}

All pregnant women are given a special set of instructions to inform the research team regarding all hospital admissions during pregnancy. All admission related data will be obtained through a surveillance system set up for this purpose. Details of hospital admissions will be obtained from patient records and also through an interview during a special visit. In the third trimester, we will set up full time research assistants in two major hospitals (in which $90 \%$ of all deliveries in the area) to collect all hospital data and a small mobile team of researchers to collect data from small hospitals. A cord blood sample will be obtained from a sub-sample to assess the cord blood biochemistry as an intermediate variable for predictor and neonatal/infant outcome variables for a long-term follow-up. Data on the mode of delivery, maternal complications, birth weight, neonatal complications and establishment of breastfeeding will be collected by trained pre-intern medical officers.

\section{Fourth follow- up visit (postpartum)}

The pregnancy outcome and the neonatal outcome details will be complemented from the pregnancy record B portion and Child Health Development Record (CHDR) B portion, which are available with PHMs. Post partum depression will be assessed using EPDS 2 weeks after the delivery. This procedure is a part of routine care programme and will be done by PHMs. Social capital will be assessed using LSCAT- MH. Details on breastfeeding and neonatal complications will be further collected through PHMs.

\section{Fifth follow-up (infants at 1 year)}

The follow-up data after 1 year will be collected using the CHDR B portion available with the PHMs. The main focus would be the growth and development data.

\section{Laboratory procedures}

Sample analysis will be done in the Public Health Research Laboratory of the Department of Community Medicine, Faculty of Medicine and Allied Sciences, Rajarata University of Sri Lanka. All analysis will be done using a fully automated biochemistry analyser, according to the manufacturers guidelines. A fully qualified medical laboratory technician will be recruited for this purpose and internal and external quality assurance will be done as recommended for all tests.

\section{Qualitative exploration of social determinants of maternal health}

In-depth qualitative studies will be conducted using relevant subsamples of participants to understand the real life situations and the dimensions of health behaviours related to abortion, antenatal depression and anxiety, maternal deaths and near misses. Identification of participants for the studies will be based on the baseline and follow up data collected in the cohort. In-depth interviews will be conducted according to standard protocols by trained interviewers and qualitative and social science experts in the team. We will be using narrative medicine approaches to understand the meaning, perspective and patient predicament to supplement the quantitative data on building an explanatory model to mental health issues in pregnancy related to health as well as social issues. Thematic analysis, mapping, narrations and explanatory model building will be used to identify the intended social mechanisms.

\section{Data management and analysis}

The data will be available only for investigators and will be stored in password-protected computers accessible only to the investigators. A cloud-based, real-time data entry programme will be used for field data collection. The data entry will be optimized with field checks, data type restrictions and logical arguments imbedded in data entry programme. This database will be linked to the laboratory data through bar coding system.

Descriptive statistics will be computed using proportions and percentages to describe the baseline data. Loss to follow-up will be analysed separately to identify and describe the characteristics of these participants. EPDS analysis will be done as described in the EPDS guidebook and the threshold value of 9 will be used in this study to identify maternal depression and anxiety. Social capital score will be generated for different constructs and the constructs will be 
considered as variables. Incidence of abortions, still births, LBW, preterm deliveries, failure in exclusive breast feeding, growth faltering, common childhood illnesses and postpartum psychosis/depression will be calculated. The relative risk of poor social capital and mental health in pregnancy on the selected outcomes will be calculated with 95\% confidence limits. Development of the tool to identify those who are with high risk for perinatal depression will be done using significant predictors identified at binary logistic regression. We will develop a conceptual framework based on baseline data before fitting the model.

\section{Data quality assurance}

Data quality will be assured at field data collection by using standard protocols, training of data collectors, supervision and feedback. In databases planned random and frequency checking with systematic feedback will ensure the data quality during the study process. For qualitative components triangulation, standard techniques, respondent validation and reflexivity will be adopted to maintain trustworthiness of data.

\section{Ethical considerations}

Being a cohort study, there will be a few ethical concerns in this study. Written informed consent will be taken from pregnant mothers to participate in this study as well as to use routinely collected data for this research purpose. The participants will be informed that this research is conducted in parallel with the routine maternal and child health service and any abnormal finding will be reported to the health provider with the participant's consent. In addition, consent will be sought for the use of serum sample for screening of infections, serum cortisol level and any other future studies, that may require baseline assessment of serum. Data will be linked to the health records using a classified code, so that the confidentiality of data will be secured. Individual level data will not be presented in any form and summary data will be used in research publications and data dissemination.

The poor social capital identified through the questionnaire will need attention. As this is part of the routine duty of PHMs, we will inform the details to the PHM to follow-up these mothers as "risk" mothers (as per the guidelines in the routine maternal care programme in Sri Lanka) in their service delivery. Same procedure will be done for pregnant mothers with high EPDS score. These women will be referred to the $\mathrm{MOH}$ for a clinical interview and via the $\mathrm{MOH}$, we will refer the mothers for appropriate psychiatric and/or counselling services. Mothers who are reported as having gender-based violence will be given special attention and counselling. At present, the public health system has a "red book", which requires entering of these data and appropriate referral.

\section{Patient and public involvement and engagement (PPIE)}

The development of the protocol for RaPCo was based on a lengthy PPIE approach. Over a period of more than 2 years we have been having formal and informal discussions with pregnant women, public health midwives, medical officers of health and all health care providers to prioritize the health needs of pregnant women in Anuradhapura. We consulted pregnant women through a formal process to include social, neighbourhood and community level socio-economic and cultural determinants to this study. Proposal development was based on another PhD study, which took place over several years, and the one of the main component was to identify research priorities related to maternal health through an eye of pregnant women. We collaborate with pregnant women at group meetings to express their views on research questions. A pregnant colleague is included as a coinvestigator to look at the protocol critically. For the ethics application, pregnant women from the Anuradhapura formally reviewed patient information sheets/ consent forms and lay summary. The booklet prepared for pregnant women, which included some of the study tools were reviewed by pregnant women representing different social status and final product was edited based on the critical reviews. Beyond pregnant women, we had formal discussions with public health staff, three rounds of discussions with public health midwives, a formal meeting with medical officers of health, several meetings with medical officer maternal and child health (MOMCH), a meeting with secretory of health in North Central Province and also a meeting with consultant community physician (CCP) of the NCP. Informal discussions were held with two obstetricians working in the district and once the ethical clearance is obtained, we will have a full briefing meeting and a discussion with all consultant obstetricians to inform about the procedure.

During the research process, we will include pregnant women from different stages of gestation to get their perspectives, priorities and issues related to research problem and process. This will be done ensuring the privacy and confidentiality of participant information, at the same time empowering participants to be involved in the research process. We will include pregnant and postpartum women in preparation of public awareness programmes and public communication. As described in the methods, PPIE is embedded in this study through a social participatory research approach.

\section{Administrative concerns}

The study is planned in collaboration with the Regional Director of Health Services (RDHS) Anuradhapura with the approval of Provincial Director of Health Services. The provincial consultant community physician and the $\mathrm{MOMCH}$ are collaborators of this study. As a part of this study, we will have workshops to improve record keeping, quality of data and data analysis workshops for all public health field staff. 


\section{Discussion}

In June 2019, pretesting of instruments and pilot testing of the project was conducted. Major changes were done after the pre-test and pilot test to improve the understanding of questions and to improve the quality of data and sample collection procedures. Several rounds of discussions were held with stakeholders to make sure that the project is well understood and supported by all stakeholders. Training of public health staff was also carried out to improve the data quality in routine documentation process. This seemed necessary after the preliminary work on data quality in pregnancy records. Several data fields were included to the questionnaires to support the development and assessment of public healthcare delivery system in the district. We have already completed the recruitment process from July to September 2019.

Provided that data from a large sample will be collected in a cohort of pregnant mothers, the proposed project has branched out to explore maternal health conditions over a period of time. For instance, at the moment, separate studies have been already planned on assessing heart disease, anaemia, metabolic syndrome, fatty liver and out of pocket expenditure in sub-sample of pregnant mothers included in the study. Given the wealth of information planning to be obtained in this cohort, we believe this proposed study would lead to numerous related studies in the future.

Further, longitudinal data generated from this proposed study would aid in better understanding of the complex dynamics of health-related behaviours among pregnant mothers and their effects, interrelationships with health related outcomes in the pregnant women and new-borns. According to the reported literature, our proposed study is the largest community-based maternal and child health cohort study in Sri Lanka, which have used an extensive PPIE in the development phase of the study. Hence, the study findings would not only be more accurate, but also would yield information, which would be of greater practical significance for the service delivery to the concerned community.

\section{Supplementary information}

Supplementary information accompanies this paper at https://doi.org/10. 1186/s12884-020-03056-X.

Additional file 1. Latin American and Carribbean Household Food Security Scale (ELCSA)- Translated and adapted Sinhala version. Translated and adapted Sinhala language tool

Additional file 2. Latin American and Carribbean Household Food Security Scale (ELCSA)- Translated and adapted Tamil version. Translated and adapted Tamil language tool

\section{Abbreviations}

AAS: Abuse Assessment Screen; ADHD: Attention-Deficit/Hyperactivity Disorder; BP: Blood Pressure; CHDR: Child Health Development Record;
Cl: Confidence interval; CWC: Child Welfare Clinics; EPDS: Edinburgh PostPartum Depression Scale; EPMM: Ending Preventable Maternal Mortality; GA: Gestational Age; GBV: Gender Based Violence; ICD-MM: International Classification of Diseases for Maternal Mortality; LBW: Low Birth Weight; LMIC: Low and Middle Income Countries; LSCAT_MH: Low and middle income countries Social Capital Assessment Scale related to maternal Health; MBBS: Bachelor in Medicine, Bachelor in Surgery; MMR: Maternal Mortality Ratio; $\mathrm{MOH}$ : Medical Officer of Health; MOMCH: Medical Officer, Maternal and Child Health; OGTT: Oral Glucose Tolerance Test; PHM: Public Health Midwife; PHNS: Public Health Nursing Sister; POA: Period of Amenorrhea; PPIE: Patient and Public Involvement and Engagement; RaPCo: Rajarata Pregnancy Cohort; RDHS: Regional Director of Health Services; SDG: Sustainable Development Goal; TIPI: Ten Item Personality Inventory; VDRL: Venereal Disease Research Laboratory

\section{Acknowledgements}

We acknowledge Dr. Roshan Sampath, Dr. Thejana Somathilaka, RDHS office Anuradhapura for their valuable inputs during the designing phase of this study. We also Acknowledge Dr. Bhagya Jayasiri for the corporation given in initiating the design of hospital data collection in this study.

\section{Authors' contributions}

SBA conceived the study. TCA finalized the protocol and drafted the manuscript. SBA and NDW extensively revised and edited the protocol. Following authors contributed in conception, designing and acquisition of the bellow mentioned specific components of the study; RIRP, MKLI, JMSB and PMBJ: qualitative components for social aspects of maternal deaths, abortion and psychological distress. AH, NL and SBA: heart disease in pregnancy. GA, VM, TCA: anaemia in pregnancy, IJ, AKD and SBA: metabolic syndrome in pregnancy. IK, DK, JW, JP and SBA: liver disease and gestational diabetes malletus in pregnancy. SPG, NW, RIRP and SBA: Economic and productivity cost analysis in pregnancy. SW, NW and TCA mindfulness in pregnancy. AW, GA and TCA: mental health in pregnancy. TCA, MW and SBA: Social capital in pregnancy, KM and HJ contributed in conception, designing and acquisition of data at regional level. AKD, JP, NH, IJ and SBA: contributed in conception, designing and acquisition of data of the hospital component of the study and MW, AH, NW and SBA designed and drafted the data quality assurance plan. All authors approved the final version of the manuscript and have agreed to be accountable for the authors own contributions and to ensure questions related to the accuracy or integrity of any part of the work are appropriately investigated, resolved, and the resolution documented in the literature.

\section{Funding}

This research was supported by the Accelerating Higher Education Expansion and Development (AHEAD) Operation of the Ministry of Higher Education, Sri Lanka funded by the World Bank. The funding agency has no role in the design of the study and collection, analysis, and interpretation of data and in writing the manuscript.

\section{Availability of data and materials}

The datasets generated during the current study will be available with the ERC, Faculty of Medicine and Allied Sciences, Rajarata University of Sri Lanka. Once the baseline data collection is completed, we will deposit data without personal identification in OSF platform (https://osf.io). Full data related to individual papers will also be published with papers as appropriate.

Ethics approval and consent to participate

Ethical approval for this study was obtained from the Ethics Review Committee of Faculty of Medicine and Allied Sciences, Rajarata University of Sri Lanka. The first draft was approved under the ERC/2019/07 on 3rd June 2019 and the revised protocol after the pre-test and pilot testing were approved on the 13th June 2019. Informed written consent will be obtained from all participants.

\section{Consent for publication}

Consent for publication will be obtained if we are to publish any individual person's data in any form (including any individual details, images or videos). At present individual data publication is not intended in this study. 


\section{Author details}

'Department of Community Medicine, Faculty of Medicine and Allied Sciences, Rajarata University of Sri Lanka, Anuradhapura, Sri Lanka. ${ }^{2}$ Department of Social Sciences, Faculty of Social Sciences and humanities, Rajarata University of Sri Lanka, Anuradhapura, Sri Lanka. ${ }^{3}$ Department of Humanities, Faculty of Social Sciences and Humanities, Rajarata University of Sri Lanka, Anuradhapura, Sri Lanka. ${ }^{4}$ Department of Environmental Management, Faculty of Social Sciences and Humanities, Rajarata University of Sri Lanka, Anuradhapura, Sri Lanka. ${ }^{5}$ Department of Business management, Faculty of Management studies, Rajarata University of Sri Lanka, Anuradhapura, Sri Lanka. ${ }^{6}$ Department of Anatomy, Faculty of Medicine and Allied Sciences, Rajarata University of Sri Lanka, Anuradhapura, Sri Lanka. ${ }^{7}$ Department of Physiology, Faculty of Medicine and Allied Sciences, Rajarata University of Sri Lanka, Anuradhapura, Sri Lanka. ${ }^{8}$ Department of Medicine, Faculty of Medicine and Allied Sciences, Rajarata University of Sri Lanka, Anuradhapura, Sri Lanka. ${ }^{9}$ Department of Pathology, Faculty of Medicine and Allied Sciences, Rajarata University of Sri Lanka, Anuradhapura, Sri Lanka. ${ }^{10}$ Department of Obstetrics and Gynaecology, Faculty of Medicine and Allied Sciences, Rajarata University of Sri Lanka, Anuradhapura, Sri Lanka. ${ }^{11}$ Teaching Hospital Anuradhapura, Anuradhapura, Sri Lanka. ${ }^{12}$ Regional director of Health Services Office, Anuradhapura, Sri Lanka. ${ }^{13}$ Provincial Director of Health Services Office, Anuradhapura, Sri Lanka. ${ }^{14}$ Department of Psychiatry, Faculty of Medicine and Allied Sciences, Rajarata University of Sri Lanka, Anuradhapura, Sri Lanka.

\section{Received: 30 August 2019 Accepted: 15 June 2020} Published online: 26 June 2020

\section{References}

1. Alkema L, Chou D, Hogan D, Zhang S, Moller A-B, Gemmill A, et al. Global, regional, and national levels and trends in maternal mortality between 1990 and 2015, with scenario-based projections to 2030: a systematic analysis by the UN maternal mortality estimation inter-agency group. Lancet. 2016; 387(10017):462-74.

2. Hogan MC, Foreman KJ, Naghavi M, Ahn SY, Wang M, Makela SM, et al. Maternal mortality for 181 countries, 1980-2008: a systematic analysis of progress towards millennium development goal 5. Lancet. 2010;375(9726): 1609-23.

3. World Health Organization. Strategies towards ending preventable maternal mortality (EPMM). Geneva: World Health Organization; 2015

4. Bonanni P, Bechini A, Pesavento G, Boccalini S, Tiscione E, Graziani G, et al. Implementation of the plan for elimination of measles and congenital rubella infection in Tuscany: evidence of progress towards phase II of measles control. J Prev Med Hyg. 2005:46(3):111-7.

5. Souza J, Tunçalp Ö, Vogel J, Bohren M, Widmer M, Oladapo O, et al. Obstetric transition: the pathway towards ending preventable maternal deaths. BJOG An Int J Obstet Gynaecol. 2014;121:1-4.

6. WHO. Maternal Mortality. 2016.

7. World Health Organization, UNICEF, UNFPA, Bank TW. Trends in maternal mortality: 1990 to 2010 WHO, UNICEF, UNFPA and The World Bank estimates. 1st ed. Geneva: World Health Organization; 2012. p. 59.

8. Putnam RD. Bowling alone: the collapse and revival of American community. New York: Simon and Schuster; 2001. p. 544.

9. Hanifan L. The rural school community Centre. Ann Am Acad Pol Soc Sci. 1916:67:130-8.

10. Portes A. Social capital: its origins and applications in modern sociology. Annu Rev Sociol. 1998;24(1):1-24.

11. Lamarca GA, do CLM, Sheiham A, Vettore M V. The association of neighbourhood and individual social capital with consistent self-rated health: a longitudinal study in Brazilian pregnant and postpartum women. BMC Pregnancy Childbirth [Internet]. 2013/01/18. 2013;13:1. Available from: http://www.ncbi.nlm.nih.gov/entrez/query.fcgi?cmd=Retrieve\&db= PubMed\&dopt=Citation\&list_uids=23324161.

12. Ehsan AM, Silva MJ De. Social capital and common mental disorde: a systematic review. J Epidemiol Commun Health. 2015;1021-8.

13. Kawachi I, Berkman L. Social cohesion, social capital, and health. In: Social epidemiology; 2014. p. 290-319.

14. De Silva MJ, Harpham T, Huttly SR, Bartolini R, Penny ME. Understanding sources and types of social capital in Peru. Community Dev J [Internet]. 2007:42(1):19-33 Available from: http://cdj.oxfordjournals.org/content/42/1/1 9.abstract.
15. Ahn S, Youngblut JAM. Predictors of women's postpartum health status in the first 3 months after childbirth. Asian Nurs Res (Korean Soc Nurs Sci). 2007;1(2):136-46.

16. Elsenbruch S, Benson S, Rucke M, Rose M, Dudenhausen J, PincusKnackstedt MK, et al. Social support during pregnancy: effects on maternal depressive symptoms, smoking and pregnancy outcome. Hum Reprod. 2007;22(3):869-77.

17. Kritsotakis G, Vassilaki M, Melaki V, Georgiou V, Philalithis AE, Bitsios P, et al. Social capital in pregnancy and postpartum depressive symptoms: a prospective mother-child cohort study (the Rhea study). Int J Nurs Stud. 2013;50(1):63-72.

18. Agampodi TC, Rheinländer T, Agampodi SBSB, Glozier N, Siribaddana S. Social capital and health during pregnancy; an in-depth exploration from rural Sri Lanka. Reprod Health. 2017;14(1):89.

19. Ma O, Ma O. Suicide: the leading cause of maternal death. Br J Psychiatry. 2003;183(4):279-81.

20. Gentile S. Suicidal mothers. J Inj Violence Res. 2011:3:90-7.

21. Lysell H, Dahlin M, Viktorin A, Ljungberg E, D'Onofrio BM, Dickman $P$, et al Maternal suicide - Register based study of all suicides occurring after delivery in Sweden 1974-2009. PLoS ONE. 2018;13(1):e0190133. https://doi. org/10.1371/journal.pone.0190133.

22. Yusuf HR, Akhter HH, Chowdhury ME, Rochat RW. Causes of death among women aged 10-50 years in Bangladesh, 1996-1997. J Health Popul Nutr. 2007;25(3):302-11.

23. Gelaye B, Kajeepeta S, Williams MA. Suicidal ideation in pregnancy: An epidemiological review. Arch Womens Ment Health. 2016;19(5):741-51.

24. Fisher J, Cabral de Mello M, Patel V, Rahman A, Tran T, Holton S, et al. WHO | Prevalence and determinants of common perinatal mental disorders in women in low- and lower-middle-income countries: a systematic review. Bull World Health Organ. 2012;90(2):139-149H World Health Organization.

25. Hendrick V, Altshuler L, Cohen L, Stowe Z. Evaluation of mental health and depression during pregnancy: position paper. Psychopharmacol Bull. 1998; 34(3):297-9.

26. Marcus SM. Depression during pregnancy: rates, risks and consequences-Motherisk update 2008. Can J Clin Pharmacol. 2009;16(1):e15-22.

27. Agampodi SB, Agampodi TC. Antenatal depression in Anuradhapura, Sri Lanka and the factor structure of the Sinhalese version of Edinburgh post partum depression scale among pregnant women. PLoS One United States. 2013;8(7):e69708.

28. O'hara MW, Swain AM. Rates and risk of postpartum depression-a metaanalysis. Int Rev Psychiatry. 1996;8(1):37-54.

29. Wadhwa PD, Sandman CA, Porto M, Dunkel-Schetter C, Garite TJ. The association between prenatal stress and infant birth weight and gestational age at birth: a prospective investigation. Am J Obstet Gynecol. 1993;169(4):858-65.

30. Dejin-Karlsson E, Hanson B, Ostergren P, Lindgren A, Sjoberg N, Marsal K Association of a lack of psychosocial resources and the risk of giving birth to small for gestational age infants: a stress hypothesis. BJOG [Internet]. 2000; 107(1):89-100 Available from: http://www.ncbi.n/m.nih.gov/entrez/query. fcgi?cmd=Retrieve\&db=PubMed\&dopt=Citation\&list_uids $=10645867 \% 5$ Cnpapers2://publication/uuid/B52B2612-CAF3-4C93-9E2C-3A04656853C5.

31. Hashim TJ, Moawed SA. The relation of low birth weight to psychosocial stress and maternal anthropometric measurements. Saudi Med J. 2000;21(7): 649-54.

32. Bernard-Bonnin A-C. Maternal depression and child development. Paediatr Child Health. 2004;9(8):575-98.

33. Cohn JF, Tronick E. Specificity of infants' response to mothers' affective behavior. J Am Acad Child Adolesc Psychiatry. 1989;28(2):242-8.

34. Tronick E, Reck C. Infants of depressed mothers. Harv Rev Psychiatry. 2009; 17(2):147-56.

35. Singer J, Fagen J. Negative affect, emotional expression, and forgetting in young infants. Dev Psychol. 1992;28(1):48-57.

36. Hammen C, Burge D, Burney E, Adrian C. Longitudinal study of diagnoses in children of women with unipolar and bipolar affective disorder. Arch Gen Psychiatry. 1990;47(12):1112-7.

37. Lesesne CA, Visser SN, White CP. Attention-deficit/hyperactivity disorder in school-aged children: association with maternal mental health and use of health care resources. Pediatrics. 2003;111(5):1232-7.

38. Faisal-Cury A, Menezes PR. Antenatal depression strongly predicts postnatal depression in primary health care. Rev Bras Psiquiatr. 2012;34(4):446-50.

39. Leigh B, Milgrom J. Risk factors for antenatal depression, postnatal depression and parenting stress. BMC Psychiatry. 2008;8:24. 
40. Rahman A, Creed F. Outcome of prenatal depression and risk factors associated with persistence in the first postnatal year: prospective study from Rawalpindi, Pakistan. J Affect Disord. 2007;100(1-3):115-21.

41. World Health Organization, Organization WH. The WHO application of ICD10 to deaths during pregnancy, childbirth and puerperium: ICD-MM. Geneva: World Health Organization; 2012. p. 1-67.

42. Agampodi S, Wickramage K, Agampodi T, Thennakoon U, Jayathilaka N, Karunarathna D, et al. Maternal mortality revisited: the application of the new ICD-MM classification system in reference to maternal deaths in Sri Lanka. Reprod Health England. 2014;11(1):17.

43. Agampodi TC, Agampodi SB, Wickramasinghe WA, Adhikari AM, Chathurani HK. Post partum depression - a problem that needs urgent attention. Ceylon Med J. 2011;56(4):183-4.

44. Family Health Bureau. MCH statistics 2016. 2018.

45. Medical Statistics Unit Ministry of Health Nutrition and Indigenous Medicine. Annual Health Statistics 2016. Colombo: Ministry of Health, Nutrition and Indigenous Medicine; 2016.

46. Department of Census and Statistics Ministry of National Policies and Economic Affairs. Sri Lanka Demographic and Health Survey 2016. Colombo; 2017.

47. Grote NK, Bridge JA, Gavin AR, Melville JL, lyengar S, Katon WJ. A metaanalysis of depression during pregnancy and the risk of preterm birth, low birth weight, and intrauterine growth restriction. Arch Gen Psychiatry. 2010; 67(10):1012-24.

48. Kelsey JL. Cohort studies. J Rheumatol Suppl. 1983;10:96-9.

49. Rowel D, Jayawardena P, Fernando N. Validation of the Sinhala translation of Edinburgh postnatal depression scale. Ceylon Med J. 2008:53(1):10-3.

50. Parker B, McFarlane J. Identifying and helping battered pregnant women. MCN Am J Matern Child Nurs. 1991;16(3):161-4.

51. Agampodi S, Agampodi T, Wickramasinghe N, Fernando S, Chathurani U, Adhikari W, et al. Productivity cost due to maternal ill health in Sri Lanka. PLoS One. 2012;7(8):1-5 Public Library of Science.

52. Gosling SD, Rentfrow PJ, Swann WB. A very brief measure of the big-five personality domains. J Res Pers. 2003;37(6):504-28.

53. Agampodi TC, Agampodi SB, Glozier N,et al. Development and validation of the Social Capital Assessment Tool in pregnancy for Maternal Health in Low and middle income countries (LSCAT-MH). BMJ Open. 2019;9:e027781. https://doi.org/10.1136/bmjopen-2018-027781

54. Abeysena H, Jayawardana P, Peiris U, Rodrigo A. Validation of the Sinhala version of the 12-item general health questionnaire. J Postgrad Inst Med. 2014;1(1):1-7.

55. Perez Escamilla R, Dessalines M, Finnigan M, Pacho H. Household Food Insecurity Is Associated with Childhood Malaria in Rural Haiti 1 , 2. 2009;(7).

56. Sumathipala A, Murray J. New approach to translating instruments for crosscultural research: a combined qualitative and quantitative approach for translation and consensus generation. Int J Methods Psychiatr Res. 2000;9(2): 87-95.

57. Department of census and Statistics. Literacy Rates by District and Sex (Population of Age 10 Years and Over).

58. World Health Organization. Waist circumference and waist-hip ratio: report of a WHO expert consultation, Geneva, 8-11 December 2008. Geneva; 2011.

\section{Publisher's Note}

Springer Nature remains neutral with regard to jurisdictional claims in published maps and institutional affiliations.

\section{Ready to submit your research? Choose BMC and benefit from:}

- fast, convenient online submission

- thorough peer review by experienced researchers in your field

- rapid publication on acceptance

- support for research data, including large and complex data types

- gold Open Access which fosters wider collaboration and increased citations

- maximum visibility for your research: over $100 \mathrm{M}$ website views per year

At $\mathrm{BMC}$, research is always in progress.

Learn more biomedcentral.com/submissions 